International Journal of Engineering \& Technology, $7(2)(2018) 506-511$
International Journal of Engineering \& Technology
SPC
Website: www.sciencepubco.com/index.php/IJET
doi: $10.14419 /$ ijet. $v 72.8428$
Research paper

\title{
Design and simulation of a 2DOF PID controller based on particle swarm optimization algorithms for a thermal phase of hybrid vehicle
}

\author{
Mohamed Elhedi Hmidi ${ }^{1}$, Ines Ben Salem ${ }^{1}$, Lilia El Amraoui ${ }^{1}$ \\ ${ }^{1}$ Research Unit in Mechatronics System and Signals (S.M.S)UR13ES49, National Engineering School of Carthage (ENICarthage) \\ University of Carthage, BP 45, Rue des Entrepreneurs, 2035 Charguia II, Tunisia \\ *Corresponding author E-mail: hmidi.med1@gmail.com
}

\begin{abstract}
This paper deals with the systematic design of a PID regulators with two degree of freedom 2DOF for a Hybrid vehicle Driving cycle based on different variants of the Particle Swarm Optimization (PSO) algorithm. The PID 2DOF problem for the stabilization of the velocity dynamics of the hybrid vehicle are formulated as a constrained optimization problem and solved thanks to improved PSO algorithms. Both PSO algorithm with variable inertia weight (PSO-In), PSO with Constriction factor (PSO-Co), PSO with possibility updating strategies (PSO-gbest) are proposed. Such variants of the PSO algorithm aim to further improve the exploration and exploitation capabilities of such a stochastic algorithm as well as its convergence fastness. All optimized 2DOF PID controllers are then simulated within a Matlab Simulink. Demonstrative simulation results are presented, compared and discussed in order to improve the effectiveness of the proposed PSO-based 2 DOF controllers for the hybrid Vehicle velocity stabilization.
\end{abstract}

Keywords: Hybrid Vehicle; Modeling; PID Two Degree of Freedom Control; Driving Cycle; Particle Swarm Optimization; PSO with Constriction Factor.

\section{Introduction}

In recent years, research and developments related to the vehicle have attracted much attention. Improvements in the autonomy, flexibility, and adaptability of these vehicles have been proposed during the thermal phase [1], [2]. The vehicle with hybrid structure, have shown a growing interest over the last decades [3].

This vehicle hybrid consists of Electric assembly and thermal with a tilting mechanism to toggle between these phase of driving This is very useful, compared to others vehicles classical, However, these structures design bring its own problems, since the degradation in stability is usually observed in the thermal phase of driving,

The history of hybrid vehicles (HV) dates back to the late 19th century [5]. However, because of their limited autonomy, the industry has further promoted the evolution and use of this new vehicle type, taking into account the availability and price of oil Following oil price inflation and taking into account environmental constraints, a great deal of research has been carried out to market a new generation of HVs equipped with cleaner sources of renewable energy. Different approaches have been studied to model the vehicles in order to increase their autonomy while minimizing the fuel consumption of the main source.

In particular, the two modes of operation of vehicles are the thermal mode corresponding to the heat engine, this is the conventional vehicle. The pure electric mode is entirely provided by the electric motor.

Thus, a dynamic model of this type of hybrid vehicle is established thanks to the Lagrange formalism [6], [7]. We are interested in the dynamic modeling of the thermal phase based on the non- linear model of this system studied obtained around a sub model to make the system easy to control.

Control methods of these nonlinear systems have been received much attention and become one of the most important topics in this research fields,

Many control schemes such as PID classical controller [8] , TwoDegree-of-Freedom PID Controllers have been employed for the velocity control and stabilization of different structures of the driving vehicle. For a conventional PID regulator structure, there are several versions, for example, the serial and parallel structure and other structures depending on the degree of freedom. The two main objectives of this controller are the follow-up of the Setpoint and the rejection Of the load disturbances. Using the $2 \mathrm{DOF}$ structure introduces other parameters that need to be defined precisely.

The History of regulators is already long and it may be interesting to recall some important steps, the first centrifugal type regulators appeared around 1750 to regulate the speed of windmills, followed in 1788 by the famous speed controller of a steam engine Of James Watt [9], [10].

As in most existing vehicle control applications, the set-point value of the controlled variable remains constant (perturbation regulation or rejection) but must be changed. They are mainly interested in the structure of two degrees of freedom (2-DoF) of PID controller algorithms.

The PID regulators with two degrees of freedom (2-DOF) include a set-point weighting on proportional terms and derivatives. A 2DOF PID controller is capable of quickly rejecting disturbances without increasing the overshoot to the set-point level. These controllers are also useful for attenuating the influence of variations of the reference signal on the control signal. 
Specific criteria for track order of hybrid vehicles include rising and settlement time. It is difficult to simultaneously satisfy these two operating conditions using a conventional controller. Therefore, the structure of the 2-DOF regulator is expected to try to meet the objectives, say good regulation and good tracking properties. This second degree of freedom add is intended to provide greater flexibility in the design of the control system [11].

This control approach is a promising design technique that shows many applications in various engineering fields. In [12], V.M. Alfaro and R. Villanova, proposed a Model Reference Robust Tuning of PID Controllers. PID Control and the new Identification and Design Methods proposed by M.A. Johnson [13]. In [14] such a 2-DOF PID approach is applied for speed control of DC Motor.

The PID 2DOF control strategy is also widely applied in the classical and modern vehicle field, due to its control methodology. It has been applied successfully to the regulation of disturbance rejections, it also remains at a given set-point and the follow-up of the controls whose controlled variable is good at monitoring the desired value [15]

However, such a gains scheduling method of a PID 2DOF controller presents some drawbacks and limitations. Indeed, the supervisor adjusts the PID gains, to be tuned with respect to many inputs and outputs variables, the shaping of membership functions, as well as the difficult and time-consuming choice of the signals scaling factors. Additional control parameters are then introduced in this 2DOF PID approach and make difficult and more complicated the tuning of the dynamics and the performance of such designed controllers, especially for nonlinear and complex systems [17]

Another difficulty lies in the choice of the predefined ranges for the proportional, integral, and derivative gains of the PID controller that depend heavily on the controlled system [30, 32] , and the set-point weights. As a solution to these drawbacks, we propose in this paper the formulation of the 2DOF PID problem as a constrained optimization problem and its resolution thanks to an efficient metaheuristics based approach.

The hard optimization theory, summary in the Particle Swarm Optimization (PSO) approach, presents a promising solution for this kind of nonlinear control problem. All control parameters of a given 2 DOF PID control structure for the studied Thermal phase of a hybrid vehicle are tuned and scheduled based on improved variants of the conventional PSO algorithm.

The problem related to the specification of all predefined ranges of the PID gains as well as the manual adjustment of the scaling factors is eliminated by modifying the classical form of PID structures and replacing them with others easier tuning parameters.

So, in this document, such a PID 2DOF control strategy is used for controlling the velocity of the thermal phase of a hybrid vehicle, as well as the complete modeling of the traction chain also presented.

The remainder of this article is organized as follows. Section 2 presents the nonlinear mathematical model of the studied driving phase based on the Lagrange formalism. In Section 3, we present the formulation of the design problem as a constrained optimization problem of the 2DOF PID controller and provide the calculated control laws. Section 4 describes the demonstrative simulations that are performed to validate the proposed control approach and demonstrates the proposed variants of the PSO algorithm for resolving such an optimization problem. Finally, the conclusions are drawn from section 5

\section{Vehicle hybrid modeling}

\subsection{System model}

The model of the thermal phase of the hybrid vehicle is a rigid body, in this model Let us consider an Internal Combustion Engine powered Vehicle (ICEV) and let us make the hypothesis is that it is possible to measure the speed $\mathrm{v}(\mathrm{t})$ of the vehicle during a trip.

Using the fundamental principle of dynamics that is written:

$M \vec{a}=\sum \vec{F}$

Where $\vec{a}$ is the vehicle acceleration, $\vec{F}$ the sum of streaks forces on the vehicle. we notice $\vec{F}_{x}$ the tractive force abandoned the wheels supplied by the motor and $\vec{F}_{\text {ext }}$ all external forces.

So the main resistance forces are the aerodynamic force $\vec{F}_{A}$, rolling resistance $\vec{F}_{R}$ and the force of gravity $\vec{F}_{G}$. In Figure 1 , they are shown by their standards denoted respectively $F_{A}, F_{R}$ et $F_{G}$ and their directions shown by the arrows. In the case of a rise with an angle $\theta$, all those forces opposed to the direction of movement of the vehicle. The tractive force $\vec{F}_{x}$ is itself in the direction of path And each can be expressed as the follows, Aerodynamic Force is calculated as

$F_{A}=\frac{1}{2} \rho S C_{x} v_{r}^{2}$

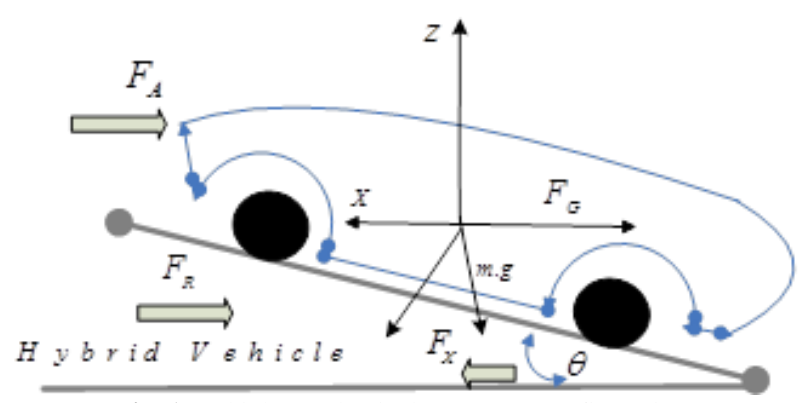

Fig. 1: Vehicle Mechanical Structure Configuration.

Rolling Resistance force is formulates using

$F_{R}=M_{g} C_{R}$

The gravity force using

$F_{G}=M g \sin (\theta)$

With $\mathrm{S}$, the frontal surface of the vehicle, $C_{x}$, the aerodynamic drag coefficient, $C_{R}$ the rolling resistance coefficient, $g$, the gravity acceleration and $\theta$, the road slope angle

\subsection{Force of the tires provided by the engine}

The conventional vehicle is only equipped with a conventional combustion engine running on gasoline. the mechanical braking device is loading slow the vehicle during braking.

The torque engine $T_{e}$ at the output of the motor shaft is delivered to the input of the gearbox via the clutch. Considering $I$, the engine rotational inertia, $w$ e the acceleration angular of engine,

So, the torque to the output of the clutch is:

$$
T_{c}=T_{e}-I_{e} \dot{w}_{e}
$$

At the output of the gearbox, the torque engine at the input is amplified by the gear ratio, then we noted

$$
\mathrm{T}_{\mathrm{d}}=\left(\mathrm{T}_{\mathrm{c}}-\mathrm{I}_{\mathrm{t}} \dot{\omega}_{\mathrm{e}}\right) \mathrm{N}_{\mathrm{t}}
$$


Where $\mathrm{N}_{t}$ the value of the transmission ratio of the box and $I_{\text {, }}$ the rotational inertia of the transmission.

At the same principle ,the torque $T_{a}$ is needed to turn the wheels and providing the transmission of force:

$$
\mathrm{T}_{\mathrm{a}}=\left(\mathrm{T}_{\mathrm{d}}-I_{\mathrm{d}} \dot{\omega}_{\mathrm{d}}\right) N_{f}
$$

Where $I_{d}$ is the rotational inertia of the drive shaft, $\dot{W}_{d}$ the angular acceleration and $N_{f}$ the differential gear ratio.

Finally combining all the above equations, the torque supplied by the engine through the transmission and given by:

$\mathrm{T}_{\mathrm{a}}=\left(\left(\mathrm{T}_{\mathrm{e}}-\left(I_{\mathrm{e}}+I_{\mathrm{t}}\right) \omega_{\mathrm{e}}\right) N_{t}-I_{d} \omega_{d}\right) N_{f}$

In the existence of the braking torque, the actual torque $T_{x}$ created by this equation:

$$
\mathrm{T}_{\mathrm{x}}=r F_{x}+I_{\omega} \dot{w}_{\omega}=T_{a}-T_{b}
$$

We conclude, the tractive force to the wheels delivered by the combustion engine can be written as:

$$
F_{x}=1 / r\left\{\eta T_{e} N-\left\{I_{e t} N^{2}+I_{d} N_{f}^{2}\right\} \dot{w}_{\omega}-T_{b}\right\}
$$

\subsection{Equation of movement}

The tractive force is knowing $F_{x}$ provided by the engine and the different forces opposing the motion of the vehicle, using the fundamental principle of dynamics, so, we get the equation of motion of the vehicle approximated by the equation:

$$
M v=F_{x}-F_{R}-F_{A}-F_{G}
$$

With the absence of longitudinal sliding, the angular velocity of the wheel is given by the equation following:

$$
\dot{v}=r \dot{w}_{\omega}
$$

Where $r$ is the wheel radius, and $w_{\omega}$ is the angular velocity of the wheel.

In conclude therefore the final equation of motion of the vehicle:

$M \dot{v}=\frac{\eta T_{e} N / r-\left\{I_{e t} N^{2}+I_{d} N_{f}{ }^{2}+I_{\omega}\right\} \dot{v}}{r^{2}}-H$

So we note:

$$
\left(M+\left(\left(I_{e l} N^{2}+I_{d} N_{f}{ }^{2}+I_{\omega}\right) / r^{2}\right)\right) \dot{v}=\left(\eta T_{e} N-T_{b} / r\right)-H
$$

Finally we find:

$$
\dot{v}=r / M r^{2}+I_{e t} N^{2}+I_{d \omega} *\left(\eta T_{e} N-T_{b}-r H\right)
$$

Where

$$
\left\{I_{d \omega}=I_{d} N_{f}{ }^{2}+I_{\omega}\right.
$$

The longitudinal modeling of the vehicle and therefore described by the following system of equations:

$$
\left\{\begin{array}{l}
\dot{x}=v_{x} \\
\dot{v_{x}}=\frac{r}{M r^{2}+I_{e r} N^{2}+I_{d \omega}} *\left(\eta T_{e} N-T_{b}-r H\right)
\end{array}\right.
$$

Where:

$$
\left\{H=M_{g} C_{R}+0.5 \rho S C_{X} v^{2}+M_{g} \sin _{\theta}\right.
$$

\section{Control design strategy}

\subsection{Proposed 2-DOF PID control structure}

Since the hybrid vehicle model thermal phase is strongly coupled and nonlinear, the proposed PID 2DOF control system is a multiobjective problem. A general form of the 2DOF PID controller is shown in Figure 2, where the controller consists of two compensators $A_{1}(s)$ and $A_{2}(s)$

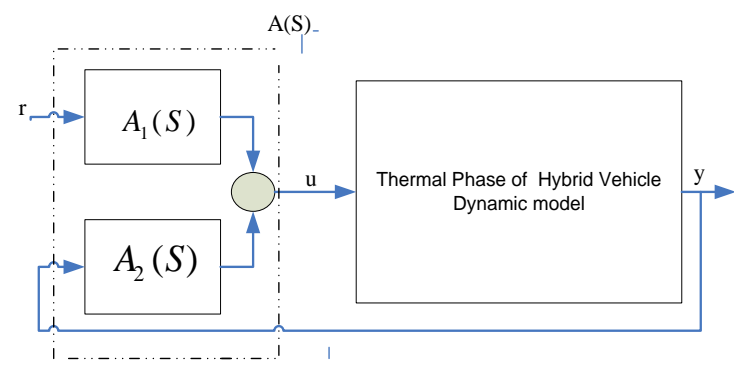

Fig. 2: 2DOF PID Controller Structure of Hybrid Vehicle.

Since the controller PID 2DOF is a controller with two inputs, an output of the form $A(s)$,as shown in the following figure. The transfer function of each input to the output is itself a PID controller. This allows us to introduce the following equations which are used to control the speed of our vehicle can be written respectively as:

$$
\begin{aligned}
& A_{1}(s)=\left(c_{1}\left(K_{p}+K_{i} / s\right)+c_{2}\left(K_{d} / T_{f}+1\right)\right) \\
& A_{2}(s)=\left(K p+K_{i} / s+K_{d} / T_{f}+1\right)
\end{aligned}
$$

Where $K_{p}, K_{i}, K_{d}$ are respectively the controller gain, the integral time constant and the derivative time, constant, $c_{1}$ and $c_{2}$ the set-point weights, and $T_{f}$ the derivative filter constant The relationship between the two inputs ( $r$ and $y$ ) and the output $(u)$ of the 2DOF PID controller can be represented in a parallel manner. The two forms are different, one can therefore express the proportional, integral and derivative actions of the controller, The Independent gains or "parallel" 2DOF PID control algorithm is expressed by the equation follows:

$$
u=K_{p}\left(c_{1} r-y\right)+K_{i} / s(r-y)+K_{d} s / T_{f}+1\left(c_{2} r-y\right)
$$

Where, $u$ is the Manipulated variable, $y$ is the controlled variable or output, and $r$ is the set-point.

\subsection{Proposed optimization problem formulation}

As described above, the tuning parameters of the proposed 2 DOF PID controllers for the Thermal phase of hybrid vehicle driving cycle are the input and output scaling factors $K_{p}, K_{i}, K_{d}$ and the set-point weights $c_{1}$ and $c_{2}$.

These design parameters present the decision variables of the following objective optimization problem, which is formulated under various operational constraints of the Thermal phase: 


$$
\left\{\begin{array}{l}
\text { minimise } f_{i}(x) \\
x=\left(k_{i}, k_{p}, k_{d}, c_{1}, c_{2}\right) \\
\text { subject to : } \\
g(x)=\delta_{v}-\delta_{v}{ }^{\max } \leq 0
\end{array}\right.
$$

Where $f_{i}: \mathbb{R}^{15} \rightarrow \mathbb{R}$ are the cost functions, $s=\left\{x \in \mathbb{R}_{+}^{15}, x_{\text {min }} \leq x \leq x_{\text {max }}\right\}$ the bounded search space of the decision variables, $g_{j}: \mathbb{R}^{15} \rightarrow \mathbb{R}$ are the problem inequality constraints. The terms $\delta_{v}$ denote the overshoots of the controlled velocity and $\delta_{v}{ }^{\max }$ is pre-specified maximum value.

The cost functions of problem (21) are chosen, for each controlled variable $v$, as the Absolute Error (IAE) criteria , defined with the generic form as follows:

$$
f_{i}(x)=\int_{0}^{+\infty}\left|e_{i}(x, t)\right| d t, i \in\{v\}
$$

Where $e_{i}(x, t)$ is the error signals between the reference and plant output taken on the step responses of the velocity $v$ To deal with constraints of problem (23), several techniques have been proposed.

One useful approach is by augmenting the cost function with penalties.

$$
\varphi(x)=f(x)+\sum_{j=1}^{\text {ncomp }} \Lambda_{j} \max \left[0, g_{i}(x)\right]^{2}
$$

Where $\Lambda_{j}$ is a prescribed scaling penalty parameters and $n_{\text {com }}$ is the number of problem constraints.

\subsection{Proposed particle swarm optimization algorithms}

\subsubsection{Overview}

The PSO metaheuristic is an evolutionary computation method developed by J. Kennedy and R.C. Eberhart [20]. This advanced global technique is inspired by the swarming behavior of biological populations [21]. In comparison with an others methods of metaheuristics, this optimization technique it is a simple concept, it is computationally efficient algorithm and easy to implement The convergence and parameters selection of this algorithm are proved [22]. PSO theory has been enormously successful in various industrial domains, but more significantly in control and optimal engineering design as shown in [21], [22].

\subsubsection{Canonical PSO algorithm}

The basic PSO algorithm uses a swarm consisting of numbers of particles $n p$, i.e., $x^{1}, x^{2}, \ldots, x^{n p}$ randomly distributed in the considered initial search space, to find an optimal solution of a generic optimization problem. Each particle, as a potential solution, is characterized by a position and a velocity given by $x_{k}^{i}:=\left(x_{k}^{i 1}, x_{k}^{i 2}, \ldots, x_{k}^{i, d}\right)^{T}$ and $v_{k}^{i}:=\left(v_{k}^{i 1}, v_{k}^{i 2}, \ldots, v_{k}^{i, d}\right)^{T}$

At each algorithm iteration, the ith particle position, $x_{i} \in \mathbb{R}^{d}$ evolves based on the following update rules:

$x_{k+1}^{i}=x_{k}^{i}+v_{k+1}^{i}$

$v_{k+1}^{i}=w v_{k}^{i}+c_{1} r_{1, k}^{i}\left(p_{k}^{i}-x_{k}^{i}\right)+c_{2} r_{2, k}^{i}\left(p_{k}^{g}-x_{k}^{i}\right)$

where $w$ is the inertia factor, $c_{1}$ and $c_{1}$ are the cognitive and the social scaling factors respectively, $r_{1, k}^{i}$ and $r_{2, k}^{i}$ are random numbers uniformly distributed in the interval $0,1, p_{k}^{i}$ is the best pre- viously obtained position of the ith particle and $p_{k}^{g}$ is the best obtained position in the entire swarm at the current iteration $\mathrm{k}$. Hence, the principle of a particle motion in the swarm is graphically shown in Fig 3 for a two dimensional design space.

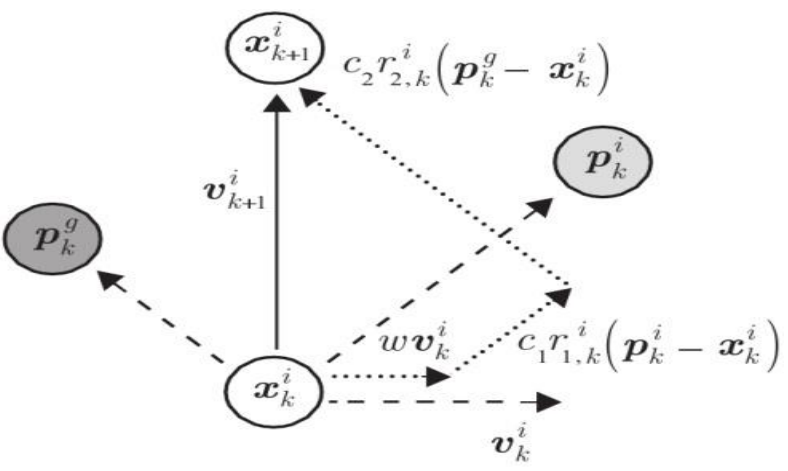

Fig. 3: Particle's Position and Velocity Update.

A one particle can be leave the search space initially defined during the motion of the swarm. So, it is should define constraints on the problem decision variables, given as the following equation

$x_{\text {min }}^{i} \leq x_{k}^{i, j} \leq x_{\text {min }}^{i}, \forall(i, j, k) \in 1, n_{p} \times 1, d \times 1, k_{\max }$

\subsubsection{PSO algorithm with inertia weight decreasing: PSO-In}

In order to improve the exploration and exploitation of the capacities of the algorithm PSO, the inertia factor of the well-known PSO-In algorithm presents a linear evolution with respect to the iterations as given for the following equation

$w_{k+1}=w_{\max }-\left(w_{\max }-w_{\min }\right) k / k_{\max }$

Where $w_{\max }=0,9$ and $w_{\min }=0.4$ are represent the maximum and the minimum inertia factor values, respectively, $k_{\max }$ is the maximum iteration number.

\subsubsection{PSO algorithm with constriction factor: PSO-Co}

In order to ensure the PSO algorithm convergence, M. Clerc introduced as a new concept of the constriction factor [22]. The velocity update equation of the algorithm PSO with Constriction factor (PSO-Co) algorithm becomes as follows:

$v_{k+1}^{i}=\chi\left\{v_{k}^{i}+c_{1} r_{1, k}^{i}\left(p_{k}^{i}-x_{k}^{i}\right)+c_{2} r_{2, k}^{i}\left(p_{k}^{g}-x_{k}^{i}\right)\right\}$

Where $\chi$ the constant is known as the constriction factor, and is given as follows

$$
\chi=2 /\left|2-\varphi-\sqrt{\varphi^{2}-4 \varphi}\right|, \varphi=c_{1}+c_{2}
$$

In [21], the authors recommended the value 4.1 for the term $\varphi=c_{1}+c_{2}$, which leads to $\chi=0.7289$, and $c_{1}+c_{2}=2.05$. Then the authors are showed that a stable convergence of the algorithm PSO-Co is guaranteed if $\varphi \geq 4$.

\subsubsection{PSO algorithm with possibility updating strategies: PSO-gbest}

This variant of the algorithm PSO is known as perturbed PSOgbest, is introduced by Z. Xinchao [18]. It is based on the perturbed gbest updating of a new strategy, which is based on the new concept of possibility measure to model the lack of information about the true optimality of the gbest $[18,19]$. This new possibility of updating strategy is characterized by a normal distribution of the global best position as follows: 


$$
p_{k}^{g} \sim \mathrm{N}\left(p_{k}^{g}, \sigma\right)
$$

Where $\sigma=\sigma(k)$ represents the degree of uncertainty about the optimality of the gbest $p_{k}^{g}$.

In the literature, it has three models have been proposed for such a possibility updating [18]. The min-max (PSO-gbest 1), linear (PSO-gbest 2) and the algorithm random (PSO-gbest 3) models are given in Eq. (31), Eq. (32) and Eq. (33), respectively:

$$
\begin{aligned}
& \sigma_{g b e s t 1}(k)=\left\{\sigma_{\max }, k<\zeta k_{\max }\right\} \\
& \sigma_{g b e r 2}(k)=\sigma_{\max }-(k-1) / k_{\max }\left(\sigma_{\max -} \sigma_{\min }\right) \\
& \sigma_{g b e r 33}(k)=\sigma_{\min }+\mu(0,1)\left(\sigma_{\max } \sigma_{\min }\right)
\end{aligned}
$$

Where $\sigma_{\max }, \sigma_{\min }$ and $\zeta$ are manually fixed parameters,

Consequently, the trajectory of each particle is updated according to its own flying experience is given by the equation (28).

\section{Control design strategies}

The control objectives of such a thermal phase of hybrid vehicle, whose physical parameters are given in Table 1, are the closedloop stabilization of its velocity

Table 1: Control Parameters of the PSO Algorithms

\begin{tabular}{ll}
\hline Parameters & Values \\
\hline Dimension of search space & $d=15$ \\
Population size & $n p=30$ \\
Number of generations & $k_{\max }=100$ \\
Cognitive coefficient & $c_{1}=0.5$ \\
Social coefficient & $c_{2}=0.5$ \\
Inertia weight & $w=0.9$ \\
\hline
\end{tabular}

Table 2: Model Parameters of the Thermal Phase Vehicle

\begin{tabular}{lll}
\hline Parameters & Description & Values \\
\hline$m$ & Mass of vehicle & $1269 \mathrm{~kg}$ \\
$r$ & Tire radius & $0.269 \mathrm{~m}$ \\
$I_{e t}$ & Motor inertia & $0.2630 \mathrm{~m}$ \\
$I_{d}$ & Inertia Drive & $0.115 \mathrm{~kg} \cdot \mathrm{m}^{3}$ \\
$I_{w}$ & Inertia of wheel & $2.8 \mathrm{~kg} \cdot \mathrm{m}^{3}$ \\
$S C_{X}$ & Aerodynamic & 0.725 \\
$\rho$ & Air density & 1.205 \\
$C_{R}$ & Rolling resistance & 0.020 \\
$\tau$ & & $R=3.72$ \\
$g$ & box report & \\
\hline
\end{tabular}

The inputs of the model are the setpoint, the gearbox and the torque engine.

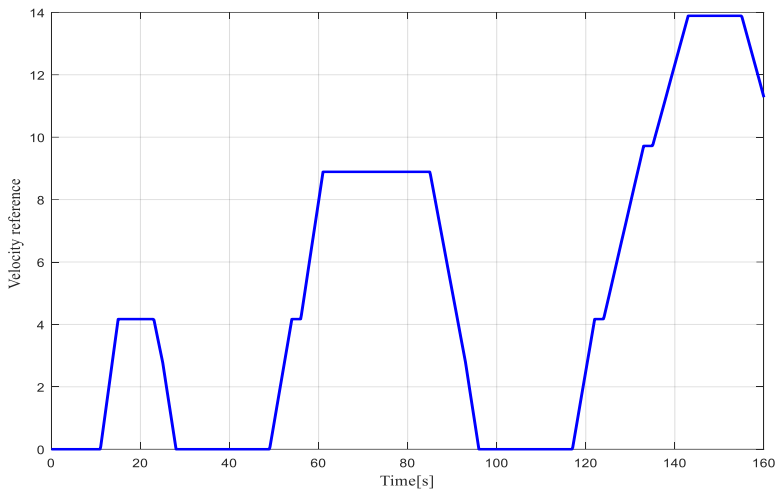

Fig. 4: NEDC Drive Cycle.

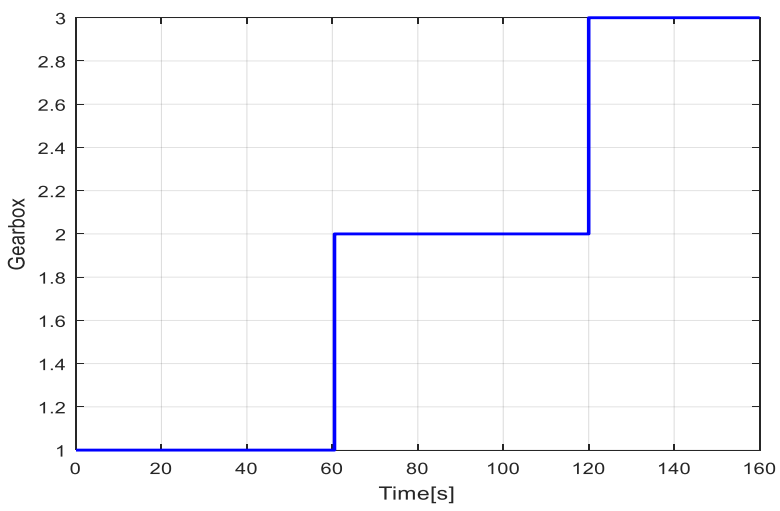

Fig. 5: The Gearbox.

Since the PSO metaheuristic is stochastic and non reproducible, all proposed PSO, PSO-In, PSO-Co and PSO-gbest algorithms are run over 30 independent times and the obtained statistical results are summarized in Table III. We denote by Best, Mean, Worst and STD the best, average, worst and standard deviation results, respectively. The CPU computation time for each optimization experiment is also given

Table 3: Optimization Results Over 30 Runs of Problem.

\begin{tabular}{lllll}
\hline Algorithms & Best & Mean & Mean & STD \\
\hline PSO & 0.01403 & 0.01436 & 0.01490 & $3.540 \mathrm{e}-04$ \\
PSO -Co & 0.01404 & 0.01421 & 0.01432 & $9.880 \mathrm{e}-05$ \\
PSO - In & 0.01370 & 0.01419 & 0.01472 & $3.631 \mathrm{e}-04$ \\
PSO - gbest 1 & 0.01440 & 0.01476 & 0.01497 & $1.816 \mathrm{e}-04$ \\
PSO - gbest 2 & 0.011479 & 0.01541 & 0.01616 & $5.551 \mathrm{e}-04$ \\
PSO - gbest 2 & 0.01538 & 0.01577 & 0.01606 & $3.140 \mathrm{e}-04$ \\
\hline
\end{tabular}

This result shows the superiority in terms of exploration and exploitation capabilities, solutions quality and fastness convergence of the PSO variants.

All proposed PSO algorithms produce 2DOF PID controllers that stabilize the velocity dynamics of the thermal phase of a hybrid vehicle as shown in Figure 5 ,

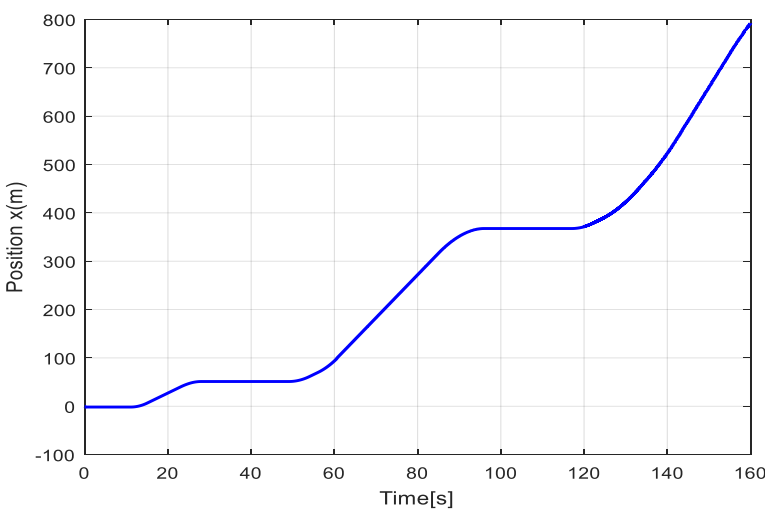

Fig. 6: The Position of Hybrid Vehicle. 


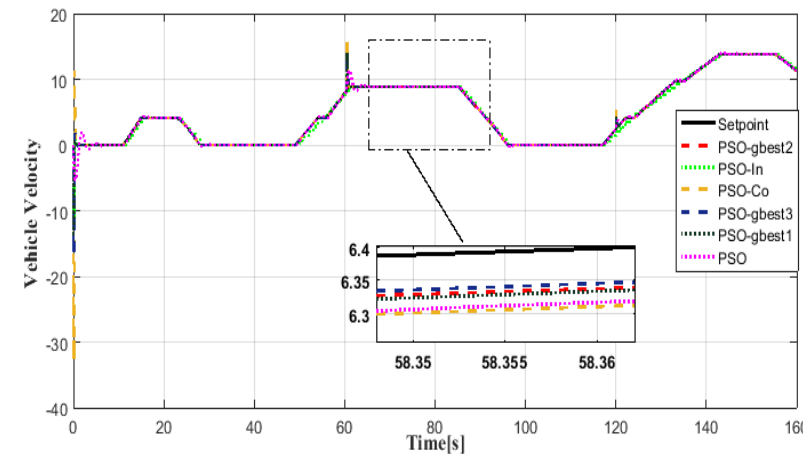

Fig. 7: Velocity of Hybrid Vehicle.

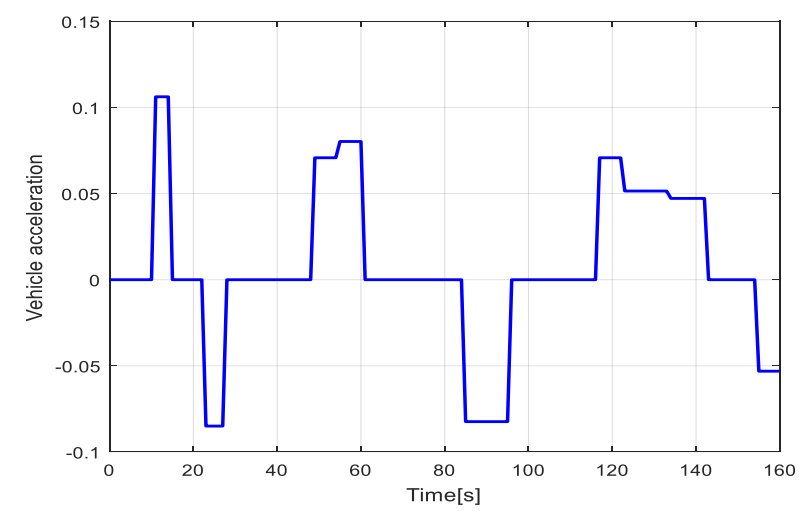

Fig. 8: The Acceleration of Hybrid Vehicle.

The simulation results of Fig. 5, 6 and Fig. 7 show the controlled position, velocity and acceleration dynamics as well as the thermal phase of hybrid vehicle powertrain control inputs. High performances in transient response and steady-state precision are guaranteed. The main purpose of such a controller is the follow-up of a NEDC Drive Cycle of hybrid vehicle and the rejection of disturbances, in our case a simulation carried out PID 2-DOF controller. Analysis and simulation results show that the new approach with PID 2-DOF Based in PSO-gbest control has wide-ranging results and performance. It is clear that the system with PSO-gbest 3 is efficient because it has a much lower overrun and good rejection of load perturbation with a minimum Stabilization time.

\section{Conclusion}

In this paper, an optimization based approach for simulated and design of a PID 2DOF controller is proposed and successfully applied for the velocity stabilization of a thermal phase of hybrid vehicle. The 2DOF PID problem for the established nonlinear model of hybrid vehicle is formulated as a constrained optimization problem and solved thanks to the proposed variants of the PSO algorithm. such advanced variants of the PSO algorithm, with variable inertia weight (PSO-In), with constriction factor (PSO-Co), and with possibility updating of the new strategies ( PSO-gbest), showed superiority, exploration and exploitation capabilities and convergence fastness of the proposed control strategy. This new design of PID 2-DOF controller is designed for the stabilization of the velocity of the hybrid vehicle. All obtained results show the effectiveness of the proposed control PID 2-DOF methodology and make easy the practical realworld implementation of the designed driving controllers .

\section{References}

[1] F. Mensing, R. Trigui, E. Bideaux (2011). «Vehicle Trajectory Optimization for Application in Eco-Driving», IEEE Vehicle Power and Propulsion Conference, September 2011, Chicago, Illinois https://doi.org/10.1109/VPPC.2011.6042993.

[2] A. Sciarretta, M. Back and L. Guzzella .Optimal Control of Parallel Hybrid Electric Vehicles, IEEE Transactions on Control Systems
Technology, Vol. 12, no. 3 , May 2004. https://doi.org/10.1109/TCST.2004.824312.

[3] M.G. Hinton, L. Forrest, D.P. Duclos, T.H. Davey, R.R. Sheahan, and K.B. Swan. Survey of driver aided devices for improved fuel economy. TechnicalReport DOT-TSC-OST-76-45, Department of Transportation, 1976

[4] ACEA (European Automobile Manufacturers' Association). Reducint $\mathrm{CO} 2$ emission: Working together to achieve better results, 2013

[5] R. Trigui, B. Jeanneret, F. Badin, "Systemic modeling of hybrid vehicles in order to predict dynamic performance and energy consumption. Building the VEHLIB library of models", (text in French with abridged version in English), RTS, November 2003

[6] T. D. Gillespie. Fundamentals of Vehicle Dynamics. Society of Automotive Engineers (SAE), 1992. https://doi.org/10.4271/R-114.

[7] J. A. Romero, AA. Lozano-Guzman, E. Betanzo-Quezada, G.M. Arroyo-Conteras, Cargo securement methods and vehicle braking performance, International Journal of Vehicle Performance, Vol. 4 ,pp. 353-373 , 2016 https://doi.org/10.1504/IJVP.2016.079919.

[8] E. Tekin , S. Ertugrul, Multi-input multi-output intelligent modeling techniques ans application to human driver, International Journal of Vehicle Performance, Vol. 4 ,pp. 390-417 , 2016 https://doi.org/10.1504/IJVP.2016.079921.

[9] J. G. Ziegler and N.B. Nichols, Optimum setting for automatic controllers, Trans ASME, Vol 64, pp. 759-768, 1942

[10] R. Gorez, New desing relations for 2-DOF PID-like control systems. Automatica, Vol. 39, pp. 901-908. https://doi.org/10.1016/S00051098(03)00029-3.

[11] k. J. Åström and T. Hägglund, Automatic tuning of simple regulators with specifications on phase and amplitude margins, Automati$c a$, vol. 20, pp. 645-651, 1984. https://doi.org/10.1016/00051098(84)90014-1.

[12] Alvaro R. Vilanova, Conversion formulate and performance capabilities of two-degree of freedom PID Control algorithm , 17th IEEE International Conference on Emerging Technologies ans factory Automation (ETFA), pp. 17-21, Karakow, Poland, sept 2012.

[13] M.A. Johnson, PID Control New Identification and design methods chap. PID Control Technology, Springer verlag London Ltd., pp. 1-46 U.K. 2005

[14] M.Kumar, P. Vandana, V. Patel, Two Degree of Freedom PID Controller For speed control of DC Motor, American International Journal of Research in Science, Technology, Engineering \& Mathematics, Vol. 39, pp. 94-97, 2015.

[15] M. Araki, Two degree of freedom PID Controllers their functions and optimal tuning, IFAC Digital Control: Past, Present and Futur of PID Control, pp. 5-7. Terrassa, Spain 2000

[16] M.Araki , H. Taguchi, Two- degree of freedom PID Controllers, International Journal of Control, Automation, and Systems, Vol. 1, No. 4, December 2003.

[17] Z-Y. Zhao, M. Tomizuka and S. Isaka. Fuzzy Gain Scheduling of PID Controllers, IEEE Transactions on Systems, Man, and Cybernetics, 1993, vol. 23, no. 5, pp. 1392-1398. https://doi.org/10.1109/21.260670.

[18] Z. Xinchao. A perturbed particle swarm algorithm for numerical optimization, Applied Soft Computing, 2010, vol. 10, pp. 119-124. https://doi.org/10.1016/j.asoc.2009.06.010.

[19] R. C. Eberhart, Y. Shi. Particle Swarm Optimization: Developments, Applications and Resources, Proceedings of the IEEE Congress on Evolutionary Computation, Seoul, Korea, 2001, pp. 81-86.

[20] R. C. Eberhart, J. Kennedy. A New Optimizer Using Particle Swarm Theory, Proceedings of the 6th International Symposium on Micro Machine and Human Science, Nagoya, 1995, pp. 39-43. https://doi.org/10.1109/MHS.1995.494215.

[21] M. L. Derouiche, S. Bouallègue, J. Haggège and G. Sandou. LabVIEW Perturbed Particle Swarm Optimization Based Approach for Model Predictive Control Tuning, Proceedings of the 4th IFAC International Conference on Intelligent Control and Automation Sciences (IFAC ICONS' 2016), Reims, France, 2016, pp. 359-364.

[22] M. Clerc and J. Kennedy. The Particle Swarm-Explosion, Stability, and Convergence in a Multidimensional Complex Space, IEEE Transactions on Evolutionary Computation, 2002, vol. 6, no. 1, pp. $58-73,2002$. 